one case occurred in the present series, and a patient of mine, a girl aged 16, developed the syndrome following irradiation of the neck for Hodgkin's disease.

The present authors point out that the early concepts of moyamoya disease as a relatively benign disorder in Japanese girls have been modified in recent reports. The sexes were equally affected and the majority of patients were Caucasian in their series. The clinical course depends on the rapidity and extent of vascular occlusion and the ability to develop a collateral circulation. The progressive nature and serious sequelae of cases left untreated are stressed.

\title{
ARTERIOVENOUS MALFORMATIONS OF THE BRAIN
}

Twenty-three children with A-V malformations have been treated by neurosurgeons at the Children's Hospital of Eastern Ontario, Ottawa, Ontario. Fourteen were boys and $\bar{g}$ giris. The averaje age at presentation was 10 years. The majority (83\%) presented with spontaneous hemorrhage and only one (4.3\%) with seizures. Angiography was performed in 21 patients. The AVM could not be demonstrated in 5 (24\%) who had an occult or cryptic AVM. Contrast-enhanced CT also failed to show abnormal vessels in 3 of the 5 occult AVMs.

Of 18 survivors, 15 were normal and 3 slightly disabled (2 with epilepsy). Aggressive surgical intervention resulted in improved survival and low morbidity. Overall mortality in the group was $22 \%$ while complete surgical excision carried a $7 \%$ mortality. The authors conclude that a spontaneous cerebral hemorrhage in a child is probably due to a vascular malformation, even when angiography and enhanced CT are negative. CT contrast enhancement is not a reliable indicator of occult AVM and direct surgery is needed for diagnostic confirmation and prevention of further hemorrhages. Also, children presenting with symptoms other than hemorrhage (e.g. seizures), should undergo surgery to prevent bleeding, providing the lesion is accessible with low risk to healthy brain. (Ventureyra ECG, Herder S. Child's Nerv Syst $1987 ; \underline{3}: 12-18$ ).

COMMENT: AVMs that bleed usually require neurosurgical management, and total excision seems the treatment preferred when feasible. Stereotaxic radiosurgery and the proton beam are available in some centers for cases not amenable to excision. The treatment of the AVM presenting with seizures or recurrent headaches but without spontaneous hemorrhage is often a neurologist's responsibility. When should he involve his neurosurgical colleague? In the past, neurologists have sometimes chosen conservative management. The present study argues in favor of surgery for all AVMs in children diagnosed radiologically, given accessibility and low risk to surrounding brain tissue. Even the neonate with the AVM that involves the vein of Galen and presents with congestive heart failure has a better chance of survival with surgery. (see HOFFMAN et al J Neurosurg $1982 ; 57: 316)$.

\section{CNS NEOPLASMS}

\section{PINEAL REGION TUMOURS OF CHILDHOOD}

At the University Hospital, Hamburg, from 1980-85, 17 children had pineal region tumours among 102 children with CNS tumours. The authors conclude: (1) that the incidence in Germany is higher than assumed and at least equal to that in Japan, (2) radiation without 
histological confirmation is not justified, (3) surgical $r$ To, 7 , successful in 10 cases, is reasonably safe, (4) cranio-spinal axis radiation, either post-operatively or alone, is indicated for: germinomas that have a tendency for CSF ependymal proliferation anci/ur seeding, (5) all children treated successfully by both surery an radiation have not relapsed. (Schulte FJ et al.EUR J Pediztr $1987 ; 146: 233-245)$.

COMMENT: Pineal tumours are of 2 main types: (1) germinomas of the pineal or suprasellar region, and (2) pineal parenchymatout tumours (pineoblastomas in younger children and pineocytomas in ol $x_{x x}$ children and adults). Spinal seeding, more common with germinomas, occurs in $2-15 \%$ of pineal tumours.

Parinaud's syndrome (impairments of upward caze, convergence- anci accommodation) is the classical presentation, usually with signs of increased intracranial pressure. Visual loss, diabetes insipidus, precocious puberty, and emaciation point to anterior hypothalamic involvement. Precocious puberty only occurs in males. Suprasellar teratomas present with visual loss, hypopituitarism, and diabetes insipidus.

Calcification occurs in 25-758 of pineal area tumours, rareiy in children under 10 years of age, and especially in germinoma 3 embryonal carcinomas. CT scan demonstrates location but may not differentiate type of tumour. Oncofetal antigens (human chor i., i : gonadotrophin and alpha-fetoprotein) in serum and spinal fluld. 7 . help in diagnosis and response to therapy.

Operative mortality and morbidity in the past have been ifig! . Shunt surgery and/or biopsy followed by radiotherapy has a 50-808 survival rate. The present authors make a point for surgical re and post-operative radiation. (For further references see (1) C C i n ME, Duffner PK. Brain Tumours in Children, Raven Press, NY, 1984; (.) Sano K, Matsutani M, Child's Brain, 1981; 8:81-97; (3) Raimondi AJ, Tomita T. Child's Brain 1982;9: $\overline{239-266)}$.

\section{DEGENERATTVE DISORDERS}

ALPERS' PROGRESSIVE NEURONAL DEGENERATION

Reporting from the Hospital for Sick Chilaren, Gieai otitun Street, London WCl, the authors have selected 13 cases, 10 boys and 3 girls, with progressive neuronal degeneration of childhood (PNDC) that was complicated by liver disease and confirmed at post mortem in 11. During life, PNDC may be suspected by a characteristic clinic? 1 course, abnormal liver function tests, and abnormalities of $P G$ (grossly asymmetric, very slow activity of high amplitude mixed with polyspikes), VER, and CAT (cortical and central atrophy and areas uf low density of the white matter). It is proposed that the term Pkid be reserved for a distinct syndrome characterized by normal init: 71 development followed by developmental retardation and later onset uf intractable seizures and liver degeneration, and by autosomil recessive inheritance.

Four patients received sodium valproate; 2 may have died flcm valproate toxicity although both had abnormal liver enzymes p.i $c$ to treatment. Phenytoin was probably blameless; 8 patients n..r received it and the liver pathology of fatty degeneration, necrosis, and cirrhosis was not that expected in phenytoin toxicity.

Brain pathology revealed cortical atrophy with predilection for 